\title{
Optimal signal processing algorithm at the background of non-Gaussian flicker noise
}

\author{
Alexander Parshin ${ }^{1, *}$ and Yury Parshin ${ }^{1}$ \\ ${ }^{1}$ Ryazan State Radio Engineering University, Radio Engineering Devices Department, 390005 \\ Gagarina str., 59/1, Russia
}

\begin{abstract}
The problem of receiving and processing ultra-low-power signals of information transmission systems is being solved. High requirements for energy efficiency on the one hand and a low information transfer rate allows the use of signals with a small spectrum width, including flicker noise spectral regions. A non-Gaussian flicker noise model is used based on a stochastic differential equation with a nonlinear drift coefficient. An optimal signal processing algorithm is being developed against the background of the sum of flicker noise and thermal noise based on an estimated-correlation-compensation approach. The analysis of the effectiveness of optimal signal processing against a background of non-Gaussian flicker noise and thermal noise is carried out.
\end{abstract}

\section{Introduction}

The problem of the frequency resource lack for data exchange is often encountered in modern information transmission systems. Dealing with the development of the Internet of Things systems, the number of simultaneously active devices is increasing sharply, while the requirements for data transmission speed are reduced. At the same time, increased demands are made on the energy efficiency of the information transmission system. One of the solutions is the usage of ultra-narrowband signals with a spectrum width about hundreds of hertz. Due to the extremely small width of the information signal spectrum, the transition to processing on a zero carrier will lead to a sharp increase in the influence of lowfrequency noise on the processing results.

To process the signals for the most common models of signals and interference, an estimation-correlation-compensation approach is used [1, 2]. In [3], a statistical approach to the description of the properties of fractal objects and signals is described, and a model of the flicker noise (FN) in the form of a fractal Brownian motion is presented, which has a frequency dependence of the power spectral density on the FN. An important feature is the fact that the fractal Brownian motion has a Gaussian probability distribution. At the same time, FN studies in many fields of radio electronics [8] prove a nonlinear mechanism of FN formation and, as a result, its non-Gaussian probability distribution. The results of theoretical and experimental studies of non-Gaussian FN models are given in [5-7].

* Corresponding author: parshin.a.y@rsreu.ru 
The aim of this work is to analyze the processing efficiency of a narrow-band signal in the presence of a non-Gaussian FN for various types of algorithms: optimal, linear, nonlinear. As a result of the analysis, the type of algorithm is determined in which the signalto-noise ratio (SNR) obtained as a result of processing monotonously increases with the growth of the analyzed sample volume.

\section{Flicker noise filtering algorithm}

The FN power spectral density has a characteristic behavior in the vicinity of zero frequency, which is sometimes called the "infrared catastrophe". With decreasing frequency, the spectral power density increases according by the law $S(f) \approx$ const $\times 1 / f^{\beta}$, where exponent $0,5 \ldots 0,8<\beta<1,2 \ldots 1,5$ for many problems of radio electronics. Moreover, in practice, flicker noise is a stationary random process, which complicates the development of its mathematical models. A common FN model is fractal Brownian motion (FBM), which has a power spectral density of the form $S(f) \approx$ const $\times 1 / f^{2 H+1}$, where $H$

- Hurst exponent. Most FBM generation algorithms are not recursive, which complicates the implementation of optimal signal processing algorithms.

There are also known non-Gaussian FN models defined by stochastic differential equations (SDEs) [5-7]. The advantage of these models is the recurrent nature, as well as the possibility of physical interpretation. When writing down the equations, we use the fact that the FN spectrum of the form $1 / f^{\beta}$ can be obtained by modeling noise using an SDE, in which the kinetic coefficient depends on the noise value non-linearly. As a result, the modeling system has a kinetic parameter that varies over a wide range, which forms the spectrum of the required shape. Since this SDE is nonlinear, the resulting FN has a nonGaussian probability distribution law.

In [7], FN modeling is considered as a solution to the SDE, in which the drift coefficient nonlinearly depends on the noise value:

$$
\frac{d x(t)}{d t}=-\frac{a x(t)}{1+\left(\frac{c}{b}\right)^{2} x^{2}(t)}+b \xi(t),
$$

where $t \in R=(-\infty, \infty)$ is a time, $\xi(t)$ - white Gaussian noise with intensity 1, $b=$ const $>0$. Drift coefficient $h(x)$ is odd function for which conditions are satisfied: $h(0)=0, a=\frac{d h(0)}{d x}>0$, there is a positive real value $L$ such as $|h(x)| \leq L|x|$ for all values $x$. There are determined conditions under which stationary solutions of SDEs exist and have longer correlations than with a linear function $h(x)$.

For modelling by discrete time with $\Delta t$ step we write down a model of a non-Gaussian FN by numerically integrating SDE (1), understood in the Ito sense, by the Euler method:

$$
x_{k}=x_{k-1}-\frac{a_{1} x_{k-1}}{1+a_{2} x_{k-1}^{2}}+a_{3} w_{k}, \quad k=1, \ldots, K
$$

where $w_{k}$ - independent standard Gaussian process in discrete time, $a_{1}=a \Delta t$, $a_{2}=\left(\frac{c}{b}\right)^{2}=\frac{c_{x}}{2 D_{x G}}, \quad a_{3}=b \sqrt{\Delta t}=\sqrt{2 D_{x G} a_{1}}, D_{x G}=\frac{b^{2}}{2 a}$ - Gaussian noise dispersion with 
$c=0$. Factor $a_{2}$ is measured in fractions of the inverse variance of flicker noise, therefore, for the convenience of normalization, the dependence is built on the parameter related by the relation $c_{x}=2 a_{2} D_{x G}$. To satisfy the conditions for the existence of an invariant probability distribution density, the following range is specified $0<c_{x}<2 / 3$.

In the simulation, the variance of the non-Gaussian FN was calculated $D_{x} \approx \frac{1}{K} \sum_{k=1}^{K}\left(x_{k}-\frac{1}{K} \sum_{k=1}^{K} x_{k}\right)^{3}$ and degree of excess $E \approx \frac{\frac{1}{K} \sum_{k=1}^{K}\left(x_{k}-\frac{1}{K} \sum_{k=1}^{K} x_{k}\right)^{4}}{\left[\frac{1}{K} \sum_{k=1}^{K}\left(x_{k}-\frac{1}{K} \sum_{k=1}^{K} x_{k}\right)^{2}\right]^{2}}-3$. Number of FN samples is $K=10^{6}$, number of realization $N_{s}=100$, Gaussian noise dispersion $D_{x G}=0,5, a_{1}=0,01$.

Figure 1 shows the dependences of the FN variance, the excess coefficient, and the power exponent in the expression for the spectral power density on the nonlinearity factor $c_{x}$ with $K=10^{6}, N_{s}=3000, D_{x G}=0,5, \quad a_{1}=0,01$.

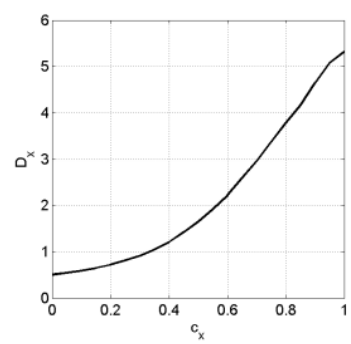

a)

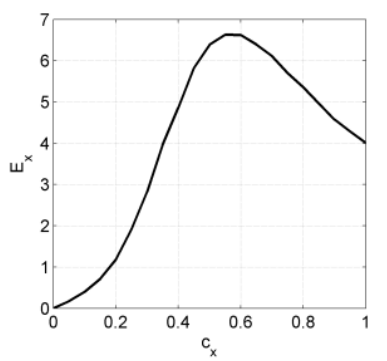

b)

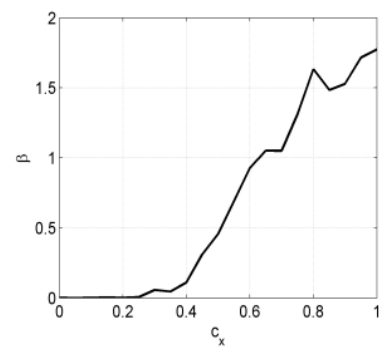

c)

Fig. 1. Statistical parameters of flicker noise.

In [8], signal processing against a FN background is based on a statistical approach. To implement the estimation-correlation-compensation (ECC) signal processing algorithm against the background of noise and noise [4], it is necessary to filter this interference not against white noise by the criterion of the minimum mean square error with following interference compensation by the resulting estimate. Since the FN model is defined as an SDE, we use the theory of filtering of Markov processes [1,9] to obtain a filtering algorithm. We set the observed process as a sum of FN $x(t)$ and white Gaussian noise $n(t)$ with density of $N_{0}: y(t)=x(t)+n(t)$. The equations for the filtering estimation and a posteriori variance are obtained by concretizing the algorithm [13] for model (1):

$$
\begin{aligned}
& \frac{d \hat{x}(t)}{d t}=-\frac{a \hat{x}(t)}{1+\left(\frac{c}{b}\right)^{2} \hat{x}^{2}(t)}+V_{x}(t) \frac{2}{N_{0}}[y(t)-\hat{x}(t)], \\
& \frac{d V_{x}(t)}{d t}=-2 a\left[\frac{1-\left(\frac{c}{b}\right)^{2} \hat{x}^{2}(t)}{\left(1+\left(\frac{c}{b}\right)^{2} \hat{x}^{2}(t)\right)^{2}}\right] V_{x}(t)-\frac{2}{N_{0}} V_{x}^{2}(t)+b^{2} .
\end{aligned}
$$


The observation equations and SDEs (2), (3) for modeling by the Euler method in discrete time are as follows:

$$
\begin{gathered}
y_{k}=x_{k}+\sqrt{D_{n}} n_{k}, \\
\hat{x}_{k}=\hat{x}_{k-1}-\frac{a \Delta t \hat{x}_{k-1}}{1+\left(\frac{c}{b}\right)^{2} \hat{x}_{k-1}^{2}}+V_{k} \frac{2 \Delta t}{N_{0}}\left(x_{k}-\hat{x}_{k-1}\right)+V_{k} \sqrt{\frac{2 \Delta t}{N_{0}}} w_{n}, \\
V_{k}=V_{k-1}-2 a \Delta t\left[\frac{1-\left(\frac{c}{b}\right)^{2} \hat{x}_{k-1}^{2}}{\left(1+\left(\frac{c}{b}\right)^{2} \hat{x}_{k-1}^{2}\right)^{2}}\right] V_{k-1}-\frac{2}{N_{0}} V_{k-1}^{2} \Delta t+b^{2} \Delta t,
\end{gathered}
$$

where $w_{k}, n_{k}$ - independent standard Gaussian processes in discrete time, $D_{n}=\frac{N_{0}}{2 \Delta t}$. During the simulation we have a parameter $p=\frac{D_{x G}}{a N_{0}}$, is equal to the ratio of the power of the Gaussian FN to the observation noise in a given frequency band $a$.

\section{Signal processing at the background of flicker noise}

Let the signal $s(t)$ be determined as a function in time. From the theory of ECC signal processing algorithm [4] it follows that at the output of the optimal FN compensator, a distorted signal is observed $\widetilde{s}(t)=s(t)+\Delta s(t)$ at the background of white Gaussian noise (innovative process) with density $N_{0}$. As a result, the signal-to-noise ratio at the output of the compensator is [1]:

$$
q=\frac{2 \overline{\widetilde{E}_{s}}}{N_{0}}=\frac{2 \int_{0}^{T} \overline{\widetilde{s}^{2}(t)} d t}{N_{0}} .
$$

Signal distortion is calculated in accordance with the expression [1] $\Delta s(t)=\hat{x}_{1}(t)-\hat{x}_{0}(t)$, in which FN estimates in absence $\hat{x}_{0}(t)$ or presence $\hat{x}_{1}(t)$ of signal are equal:

$$
\begin{gathered}
\frac{d \hat{x}_{0}(t)}{d t}=-\frac{a \hat{x}_{0}(t)}{1+\left(\frac{c}{b}\right)^{2} \hat{x}_{0}^{2}(t)}+V_{x 0}(t) \frac{2}{N_{0}}\left[y(t)-\hat{x}_{0}(t)\right], \\
\frac{d \hat{x}_{1}(t)}{d t}=-\frac{a \hat{x}_{1}(t)}{1+\left(\frac{c}{b}\right)^{2} \hat{x}_{1}^{2}(t)}+V_{x 1}(t) \frac{2}{N_{0}}\left[y(t)-\hat{x}_{1}(t)-s(t)\right] .
\end{gathered}
$$

Correspondingly, the equations for a posteriori variances $V_{x 0}(t), V_{x 1}(t)$ are also written.

We assume that the variance of the estimation error also changes slightly when a useful signal appears: $V_{1}(t) \approx V_{0}(t)$. As a result of the following simplifications, we obtain the equation for signal distortion: 


$$
\frac{d \Delta s(t)}{d t}=-\Delta s(t)\left[a \frac{1-\left(\frac{c}{b}\right)^{2} \hat{x}_{0}^{2}(t)}{\left[1+\left(\frac{c}{b}\right)^{2} \hat{x}_{0}^{2}(t)\right]^{2}}+V_{x 0}(t) \frac{2}{N_{0}}\right]-V_{x 0}(t) \frac{2}{N_{0}} s(t) .
$$

Given that the filtering error is assumed to be sufficiently small, it is possible to use the exact $\mathrm{FN}$ value instead of its estimate in the theoretical analysis of signal distortions $\hat{x}_{0}(t) \approx x(t)$.

The calculation of the signal-to-noise ratio (4) is carried out by the method of modeling signal distortions and calculating the average energy of the distorted signal:

$$
q \approx \frac{\frac{2}{N_{s}} \sum_{n=1}^{N_{s}} \sum_{k=1}^{K}(s(k \Delta t)+\Delta s(k \Delta t))_{n}^{2} \Delta t}{N_{0}} .
$$

Sufficient statistics for many signal processing problems are the logarithm of the likelihood ratio obtained by the ECC method:

$$
z=\frac{2}{N_{0}} \int_{0}^{T}\left(y(t)-\hat{x}_{0}(t)\right)\left(s_{0}+\hat{x}_{1}(t)-\hat{x}_{0}(t)\right) d t-\frac{1}{N_{0}} \int_{0}^{T}\left(s_{0}+\hat{x}_{1}(t)-\hat{x}_{0}(t)\right)^{2} d t
$$

Processing efficiency is evaluated as a signal-to-noise ratio, which is calculated from the moments of sufficient statistics:

$$
q=\frac{\bar{z}^{2}}{\overline{(z-\bar{z})^{2}}} \approx \frac{\frac{1}{N_{s}} \sum_{n=1}^{N_{s}} z_{n}}{\frac{1}{N_{s}} \sum_{n=1}^{N_{s}} z_{n}^{2}-\left(\frac{1}{N_{s}} \sum_{n=1}^{N_{s}} z_{n}\right)^{2}} .
$$

The optimal ECC algorithm (7) and several variants of quasi-optimal processing algorithms differ one from another in computational cost and efficiency.

1) Optimal ECC statistics

$$
z=\frac{2}{N_{0}} \int_{0}^{T}\left(y(t)-\hat{x}_{0}(t)\right)\left(s_{0}+\hat{x}_{1}(t)-\hat{x}_{0}(t)\right) d t-\frac{1}{N_{0}} \int_{0}^{T}\left(s_{0}+\hat{x}_{1}(t)-\hat{x}_{0}(t)\right)^{2} d t
$$

2) Non-linear FN compensation and optimal correlation processing

$$
z=\frac{2}{N_{0}} \int_{0}^{T}\left(y(t)-\hat{x}_{0}(t)\right)\left(s_{0}+\hat{x}_{1}(t)-\hat{x}_{0}(t)\right) d t .
$$

3) Nonlinear FN compensation and matched (linear) correlation processing

$$
z=\frac{2}{N_{0}} \int_{0}^{T}\left(y(t)-\hat{x}_{0}(t)\right) s_{0} d t
$$

4) Matched correlation processing

$$
z=\frac{2}{N_{0}} \int_{0}^{T} y(t) s_{0} d t
$$


Figure 2 shows the dependences of the signal-to-noise ratio (8) on samples number $K$ at various values of the nonlinearity parameter $c_{x}$. The calculation was carried out by computer modeling the equations obtained above with $a_{1}=0,01, D_{x G}=0,5, p=0,5$, $N_{s}=10^{4}, s(t)=s_{0}=0,1$. Variants of the analyzed algorithms correspond to the following numbers of the corresponding formula: (9) - «ECC», (10) - « comp+optim», (11) « comp+match », (12) - « match », (4) - « SNR».

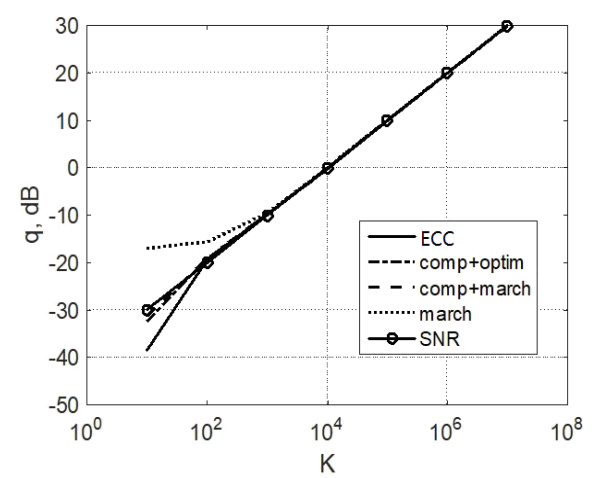

a)

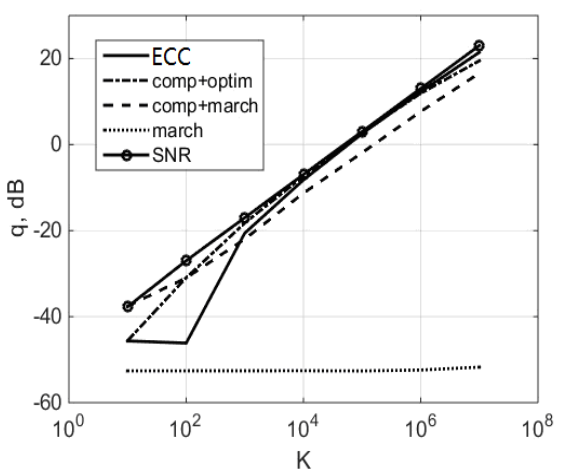

b)

$$
c_{x}=1, D_{x}=5,4, E_{x}=4, \beta=1,7
$$

Fig. 2. Dependencies of signal-to-noise ratio on samples number.

\section{Conclusion}

At the background of a Gaussian FN, the processing result is practically independent of the type of algorithm, since for the selected type of narrow-band low-frequency signal, the FN spectrum is almost constant in the signal frequency band. Therefore, the type of processing does not affect the signal-to-noise ratio.

An increase in the samples number on the one hand increases the energy of the signal, and on the other hand reduces the width of its spectrum. The first factor increases the signal-to-noise ratio, and the second, on the contrary, leads to a decrease in the signal-tonoise ratio. For a linear, matched algorithm, decreasing the width of the signal spectrum shifts the spectrum to the low-frequency region, where the FN is more intense, which compensates for the increase in signal energy. As a result, with increasing samples number, the efficiency of linear processing tends to a constant value, which corresponds to the previously obtained results for a Gaussian FN [8].

In non-Gaussian FN, the use of non-linear noise compensation is significant, more than $20 \mathrm{~dB}$, increases the signal-to-noise ratio. If the correction of the reference signal is used in accordance with the distortions of the useful signal in the interference canceller (10), (11), then the efficiency is increased by $5 \mathrm{~dB}$ compared with the matched processing (12). The use of the optimal algorithm (9), which takes into account the random nature of the energy of the reference signal, slightly increases the signal-to-noise ratio by $0.07 \mathrm{~dB}$. Thus, for all variants of algorithms with nonlinear FN compensation, an increase in samples number leads to a proportional increase in the signal-to-noise ratio.

The research has been carried out under the support of the project 8.2810.2017 in Ryazan State Radio Engineering University funded by the Ministry of science and high education of the Russian Federation 


\section{References}

1. Yu.G. Sosulin, V.V. Kostrov, Yu.N. Parshin, Estimation-correlation signal processing and interferences compensation (Radio Engineering, Moscow, 2014)

2. Yu.G. Sosulin, Yu.N. Parshin, Non-linear estimation of stochastic signals with algorithm parameters adaptation, Radio engineering and electronic, v. 5(31), pp. 904910 (1986)

3. R.M. Crownover, Introduction to Fractals and Chaos (Jones and Bartlett. 1995)

4. G.N. Bochkov, Yu.E. Kuzovlev, New in 1/f noise investigation, Uspekhi physicheskih nauk, v. 1(141), pp. 151-176 (1983)

5. B. Kaukalys, J. Ruseckas, Stochastic nonlinear differential equation generating $1 / f$ noise, Physical Review E 70, 020101(R) (2004)

6. B. Kaukalys, M. Alaburda, J. Ruseckas, Modeling non-Gaussian 1/f noise by the stochastic differential equations, Noise and fluctuations: 19th International Conference on Noise and Fluctuations - ICNF 2007, AIP Conf. Proc. 922. pp. 439-442 (2007)

7. Y.V. Mamontov, M. Willander, Long Asymptotic Correlation Time for Non-Linear Autonomous Ito's Stochastic Differential Equation, Nonlinear Dynamics 12, pp. 399411 (1997)

8. A.Yu. Parshin, Yu.N. Parshin, Optimal processing of narrowband signal in the presence of Gaussian and non-Gaussian flicker noise, Radioengineering, v. 5(83), pp. 161-167 (2019)

9. A. Sage, J. Melsa, Estimation Theory with Application to Communication and Control, IEEE Transactions on Systems Man and Cybernetics 1(4) (1971) 